\title{
Exposição aos fatores de risco do câncer do colo do útero na estratégia de saúde da família de Nova Iguaçu, Rio de Janeiro, Brasil
}

\author{
Exposure to risk factors of cervical cancer in the family health strategy \\ in Nova Iguaçu, Rio de Janeiro, Brazil
}

\author{
Ricardo de Mattos Russo Rafael ${ }^{1}$, Anna Tereza Miranda Soares de Moura ${ }^{2}$
}

\begin{abstract}
Resumo
O câncer do colo do útero apresenta-se como um importante problema de saúde pública no Brasil e no mundo. Dentre os cenários de assistência à saúde, a Estratégia de Saúde da Família parece ser um espaço privilegiado para o seu controle. O objetivo da pesquisa foi analisar o perfil da clientela e da utilização dos serviços de rastreamento do câncer do colo do útero na Saúde da Família de Nova Iguaçu, RJ. Trata-se de um estudo transversal a partir de inquérito domiciliar com uma amostra de 281 mulheres. Observou-se que 95,37\% das entrevistadas (IC95\% 92,90-97,84) relataram já ter realizado o exame alguma vez na vida. Apenas 33,80\% (IC95\% 28,24-39,37) delas não fizeram o exame no último ano. Percebeu-se que 79,71\% (IC95\% 74,98-84,44) o realizam como rotina de pesquisa para o câncer. Constatou-se que a Estratégia de Saúde da Família vem ultrapassando as expectativas e seguindo as recomendações acerca do quantitativo rastreio do câncer do colo uterino. Todavia, o perfil de fatores de risco e o intervalo entre os exames apontam para a necessidade de reflexão sobre possíveis lacunas na organização e qualidade dos métodos de prevenção e detecção precoce da doença.
\end{abstract}

Palavras-chave: neoplasias do colo do útero; esfregaço vaginal; saúde da família.

\begin{abstract}
Cervical cancer is a relevant public health problem in Brazil and in the world. Among the scenarios of health care, the Family Health Strategy seems to be a remarkable locus for its control. The aim of the study was to analyze the profile of clients and of cervical cancer screening usage on the Family Health Strategy of the municipality of Nova Iguaçu, Rio de Janeiro, Brazil. It's a cross-sectional study based on household survey with a sample of 281 women aged between 20 and 59 years. It was observed that $95.37 \%$ of the respondents $(95 \% \mathrm{Cl}$ 92.90-97.84) reported having done the exam once in their lives. Only $33.80 \%$ $(95 \% \mathrm{Cl} 28.24-39.37)$ of them were not given an examination last year. It was observed that $79.71 \%(95 \% \mathrm{Cl} 74.98-84.44)$ of the women perform as a routine search for the cancer. It was found that the Family Health Strategy has been surpassing expectations and following the recommendations on the quantitative screening of cervical cancer. However, the risk factors profile and the interval between examinations emphasize the need for reflection on possible gaps in the organization and quality of prevention methods and early detection of the disease.
\end{abstract}

Keywords: uterine cervical neoplasms; vaginal smears; family health.

Trabalho realizado na Universidade Estácio de Sá - Rio de Janeiro (RJ), Brasil.

'Doutorando em Ciências pela Faculdade de Ciências Médicas da Universidade do Estado do Rio de Janeiro (FCM/UERJ); Enfermeiro da Estratégia de Saúde da Família - Nova Iguaçu (RJ), Brasil.

${ }^{2}$ Doutora em Saúde Coletiva pelo Instituto de Medicina Social da UERJ; Médica do Hospital Universitário Pedro Ernesto - Rio de Janeiro (RJ), Brasil. Endereço para correspondência: Ricardo de Mattos Russo Rafael - Avenida Abílio Augusto Távora, 2.134 - Bairro da Luz - CEP: 26275-580 - Nova Iguaçu (RJ), Brasil -E-mail: ricko.mattos@hotmail.com

Fonte de financiamento: nenhuma.

Conflito de interesse: nada a declarar. 


\section{INTRODUÇÃO}

As neoplasias integram atualmente uma das quatro principais causas de mortalidade em adultos jovens no Brasil, configurando-se como um relevante problema de saúde pública no país e no mundo ${ }^{1}$. Com relação à população feminina, especial atenção vem sendo direcionada ao câncer de colo uterino devido às suas elevadas taxas de incidência $e$ mortalidade, ocupando as primeiras posições neste grupo. $\mathrm{O}$ Instituto Nacional do Câncer (INCA) ${ }^{2}$ estimou para o ano de 2010 o desenvolvimento de até 31,18 novos casos da doença para cada 100 mil mulheres, com elevação dos custos para a saúde e aumento da demanda por exames e tratamentos.

A partir de longa e aprofundada discussão, que culminou com a elaboração do Programa Nacional de Controle do Câncer do Colo do Útero (PNCCCU), o exame de colpocitologia oncótica com a técnica de Papanicolaou foi escolhido como o método de rastreio mais adequado, devido à sua boa relação custo-benefício-efetividade ${ }^{3,4}$.

A observação de uma maior vulnerabilidade em faixas etária superiores a 30 anos sugere que seja realizado 1 exame colpocitológico a cada 3 anos em mulheres entre 25 a 59 anos de idade, após a ocorrência de dois resultados negativos e consecutivos obtidos com intervalo inferior a um ano ${ }^{5,6}$. O programa traz ainda importante contribuição ao recomendar a ampliação da oferta do método na rede de saúde e a captação precoce de grupos vulneráveis, que podem ser consideradas ações capazes de promover a cura em até $100 \%$ dos casos em fases iniciais da doença ${ }^{7,8}$.

Todavia, as formas com que as ações de prevenção da doença vêm sendo desenvolvidas acabam por não contemplar de maneira igualitária todas as camadas da população. Esse aspecto tem importância diferenciada no que concerne ao câncer de colo de útero, na medida em que a ocorrência desta neoplasia sofre influência de características sociais e econômicas, uma vez que as regiões menos desenvolvidas socioeconomicamente apresentam maior incidência da doençç ${ }^{5,9}$. Este achado reforça a necessidade de maior atenção e ampliação das ações, a fim de que estes grupos mais vulneráveis possam ser contemplados.

Nesta perspectiva, parece interessante considerar as características dos diferentes grupos populacionais para o desenvolvimento de intervenções que objetivam a redução da incidência deste câncer. Estima-se que cerca de 90\% dos fatores de risco envolvidos na evolução da doença sejam externos aos seus aspectos genéticos e biológicos, tais como as carências nutricionais da mulher e presença de tabagismo, além dos relacionados às questões gineco-obstétricas, como a pluralidade de parceiros e precocidade do início da atividade sexual, presença de doenças sexualmente transmissíveis, multiparidade e o uso de anticoncepcionais hormonais orais. Todos estes aspectos precisam ser considerados na implementação de ações de prevenção e promoção de saúde local ${ }^{7,10}$.

A proposta de atenção à saúde desenvolvida a partir da criação do Programa de Saúde da Família (PSF), em 1994, atualmente reconhecido como Estratégia de Saúde da Família (ESF), traz a possibilidade de ampliação de atuação frente à problemática exposta. A ESF tem como um dos seus principais focos a transformação das práticas tradicionais de atenção à saúde, além da mudança no olhar dos profissionais, que passariam a praticar o cuidado integral ao indivíduo, avaliado como um sujeito partícipe de sua condição de saúde e meio social $^{11,12}$. Para alcançar estes objetivos a estratégia parte do princípio de delimitação territorial, com o reconhecimento da estrutura organizacional e prioridades de cada comunidade adscrita. A programação de suas atividades é realizada com base nas necessidades da clientela, visando à garantia de acesso a exames diagnósticos e laboratoriais previstos na Atenção Primária à Saúde. Neste caso, estão incluídas as técnicas de detecção precoce do câncer do colo uterino ${ }^{13}$.

O presente estudo tem como objetivo analisar a exposição aos fatores de risco para ocorrência do câncer do colo uterino e a utilização dos serviços de rastreamento da doença na clientela da ESF do município de Nova Iguaçu, Rio de Janeiro. Os resultados obtidos poderão contribuir com subsídios para a reprogramação das atividades da estratégia e a formulação de possíveis ações de deteç̧ão precoce desta neoplasia na região, que carece de estudos sobre o tema.

\section{MÉTODOS}

Trata-se de um estudo observacional, do tipo transversal, com entrevistas realizadas a partir de um inquérito domiciliar em regiões cobertas pela ESF de Nova Iguaçu. O município está localizado na região metropolitana do estado do Rio de Janeiro, uma de suas mais importantes áreas, tanto por sua elevada densidade populacional, quanto por seu desenvolvimento econômico. Como critérios de elegibilidade para a definição do território de pesquisa, foram consideradas as regiões administrativas mais próximas do centro e maior concentração de serviços de saúde, com quatro áreas selecionadas. Dentre elas, dois territórios da ESF foram eleitos por intencionalidade, considerando o tempo de implantação das equipes - até cinco anos - e pela maior cobertura - pelo menos $50 \%$ dos residentes da localidade.

A população-fonte da pesquisa compreendeu o conjunto de mulheres com idade entre 20 e 59 anos, moradoras da área selecionada, totalizando 1.724 sujeitos elegíveis. O tamanho amostral calculado foi de 272 mulheres, assumindo-se 
prevalência do evento de $30 \%{ }^{9,14}$, erro de $5 \%$, e $10 \%$ de perdas. Prevendo as perdas ocasionais em estudos por inquérito domiciliar, foi realizada uma sobre-amostra de $20 \%$, de forma que o tamanho amostral final chegou a 326 mulheres. Foi elaborada uma listagem com base no cadastramento realizado pelas equipes em toda a região, utilizando-se a técnica de amostragem sistemática para a escolha dos participantes.

As entrevistas foram realizadas por nove Agentes Comunitários de Saúde (ACS) pertencentes à equipe, sob a supervisão do pesquisador principal (autor do trabalho). $\mathrm{O}$ convite para participação no estudo foi realizado pelos entrevistadores, divididos em turnos, que compareceram aos domicílios das mulheres elegíveis, agendando as entrevistas. A aplicação dos instrumentos ocorreu em março de 2009 na residência das entrevistadas, com duração média de 15 minutos por entrevista. Destaca-se que nos casos em que o domicilio não favoreceu a relação entrevistador-entrevistado, foi permitida a sugestão de outro espaço de encontro, mais reservado. Nos casos em que as mulheres não poderiam ser encontradas no período diurno e nos dias úteis da semana, a conversa foi agendada em horário alternativo (noturno ou fim de semana). A busca era interrompida após duas tentativas de contato ou devido a mudanças de endereço. Destaca-se que as perdas representaram $13,8 \%$ do total da amostra e somente seis sujeitos selecionados recusaram participar. O número final de entrevistas realizadas foi 281, ultrapassando o previsto no cálculo amostral inicial.

As informações foram coletadas por meio de um questionário estruturado e multidimensional. Para a avaliação do perfil da amostra foram utilizadas algumas questões da Pesquisa Nacional por Amostra de Domicílios (PNAD) e os critérios de Classificação Econômica da Associação Brasileira de Empresas de Pesquisa ${ }^{15,16}$. Quanto às perguntas específicas do estudo, optou-se pela utilização do questionário Módulo Exames para detecção de câncer de colo de útero e mama e uso de hormônios, utilizado pelo INCA no Inquérito Domiciliar sobre Comportamentos de Risco e Morbidade Referida de Agravos Não Transmissíveis, excluídas as que se referiam ao câncer de mama ${ }^{17}$.

Após a construção do banco de dados e digitação dos instrumentos, procederam-se a limpeza, processamento e análises estatísticas por meio do software Stata SE 10 (Stata Corp., College Station, Estados Unidos). Uma análise descritiva foi utilizada para estimar a prevalência de exposição a fatores de risco do câncer do colo uterino, com os respectivos Intervalos de Confiança (IC95\%) calculados via método binomial exato ${ }^{18}$.

A fim de atender o cumprimento da Resolução CNS 196/96, os objetivos do estudo foram explicitados às equipes de Saúde da Família envolvidas e o projeto encaminhado ao Comitê de Ética em Pesquisa da Universidade Estácio de
Sá, obtendo autorização sob o Certificado de Apresentação para Apreciação Ética 0137.0.000.308-09. O estudo utilizou o Termo de Consentimento Livre e Esclarecido, apresentando os objetivos do trabalho ao entrevistado, e garantindo o anonimato das respondentes. Nos casos necessários, as participantes foram orientadas a procurar o serviço de Saúde da Família, sendo agendadas no próprio local consultas médicas e de enfermagem.

\section{RESULTADOS}

A Tabela 1 apresenta o perfil sociodemográfico da amostra estudada, com predominância de mulheres entre 20 e 39 anos $(60,1 \%$; IC95\% 54,4-65,9), em sua maioria de cor parda $(44,1 \%$; IC95\% 38,3-50,0) e casada ou em união consensual estável (65,1\%; IC95\% 59,5-70,7). Com relação à escolaridade, evidenciou-se que a maior parte das respondentes estudou até o oitavo ano do ensino fundamental. Houve predomínio da classe C entre as respondentes (59,1\%; IC95\% 53,2-64,8).

$\mathrm{Na}$ Tabela 2 estão dispostas as prevalências referentes ao histórico ginecológico e obstétrico das participantes do estudo. Observou-se que $56,6 \%$ (IC95\% 50,7-62,4) da população

Tabela 1. Perfil sociodemográfico da amostra de mulheres residentes na área de cobertura da Estratégia de Saúde da Família, Nova Iguaçu, 2009

\begin{tabular}{lc} 
Característica da amostra (n=281) & Prevalência (IC95\%) \\
Idade (anos) & \\
$20-29$ & $31,0(25,5-36,4)$ \\
$30-39$ & $29,2(23,8-34,5)$ \\
$40-49$ & $22,8(17,8-27,7)$ \\
$50-59$ & $17,1(12,6-21,5)$ \\
Raça/cor & \\
Branca & $29,9(24,5-35,3)$ \\
Preta & $25,3(20,1-30,4)$ \\
Parda & $44,1(38,2-50,0)$ \\
Amarela/indígena & $0,7(0,3-1,7)$ \\
Escolaridade & \\
Nenhuma & $5,3(2,7-8,0)$ \\
$1^{\text {a }}$ a 4 ${ }^{\text {a }}$ série & $38,4(21,7-44,1)$ \\
$5^{\text {a }}$ a $8^{\text {a }}$ série & $28,8(23,5-34,1)$ \\
Ensino médio & $25,6(20,5-30,7)$ \\
Ensino superior & $1,8(0,2-3,3)$ \\
Situação conjugal & \\
Casada/união consensual & $65,1(59,5-70,7)$ \\
Solteira & $21,7(16,8-26,5)$ \\
Desquitada/divorciada/viúva & $13,2(9,2-17,1)$ \\
Classe econômica & \\
B & $6,8(3,8-9,7)$ \\
C & $59,1(53,3-64,8)$ \\
E & $32,4(26,9-37,9)$ \\
\hline & $1,8(0,2-3,3)$ \\
\hline
\end{tabular}

IC: intervalo de confiança 
estudada teve sua menarca a partir dos 12 anos de idade. Quanto ao tempo de utilização dos anticoncepcionais hormonais orais, nota-se que um número significativo de mulheres (40,2\%; IC95\% 32,9-47,6) o fizeram no período máximo de até quatro anos, embora se perceba que 31,6\% (IC95\% $24,6-38,6)$ fizeram uso prolongado por mais de 10 anos.

$\mathrm{O}$ estudo evidenciou que a maioria das mulheres iniciou seu período de climatério com menos de 48 anos de idade (53,6\%; IC95\% 40,1-67,0) e que 19,3\% (IC95\% 8,7-29,9) utilizam terapia de reposição hormonal há 3,3 anos (desvio-padrão: 1,7-4,9). Houve maior frequência de multigestas (43,4\%; IC95\% 37,6-48,2) e quase metade das mulheres teve sua primeira gravidez abaixo dos 19 anos (47,4\%; IC95\% 41,3-53,4), o que revela grande número de gestações na adolescência.

Aspectos relacionados à realização de exames para detecção do câncer do colo uterino estão representados na Tabela 3. A maior parte das entrevistadas (95,4\%; IC95\% 92,9-97,8) relatou já ter realizado o exame colpocitológico alguma vez na vida e apenas 33,8\% (IC95\% 28,2-39,4) destas mulheres não o haviam feito no último ano. Quanto ao tempo do último exame, observou-se predomínio em intervalos menores de 1 ano (72,4\%; IC95\% 66,9-77,9), com realização, em sua maioria, no Sistema Único de Saúde (SUS) (72,0\%; IC95\% 66,6-77,4).

Quanto aos aspectos motivadores pela procura da realização da colpocitologia oncótica, observou-se que 20,28\% (IC95\% 15,55-25,01) das mulheres o fizeram devido a algum

Tabela 2. Perfil ginecológico e obstétrico das mulheres residentes na área de cobertura da Estratégia de Saúde da Família, Nova Iguaçu, 2009

\begin{tabular}{lcc} 
Característica & $\mathbf{n}$ & Prevalência (IC95\%) \\
Idade da menarca (anos) & 281 & \\
$\quad<12$ & & $43,4(37,6-49,7)$ \\
$\quad \geq 12$ & & $56,6(50,7-62,4)$ \\
Tempo de anticoncepção oral (anos) & 174 & \\
$\quad<1$ & & $9,2(4,8-13,5)$ \\
$1-4$ & & $31,0(24,1-38,0)$ \\
$5-9$ & & $28,2(21,4-34,9)$ \\
$\quad \geq 10$ & & $31,6(24,6-38,6)$ \\
Idade da menopausa (anos) & 56 & \\
$\quad<48$ & & $53,6(40,1-67,0)$ \\
$\quad \geq 48$ & & $46,4(32,9-59,9)$ \\
Situação obstétrica & 281 & \\
$\quad$ Nuligesta & & $10,0(6,4-13,5)$ \\
Primigesta & & $17,4(13,0-21,9)$ \\
Secundigesta & & $29,2(23,8-34,5)$ \\
Multigesta & & $43,4(37,6-48,2)$ \\
Idade da primeira gestação (anos) & 266 & \\
$\quad<16$ & & $10,1(6,5-13,8)$ \\
$16-19$ & & $37,2(31,4-43,1)$ \\
$20-29$ & & $42,1(36,1-48,1)$ \\
$30-39$ & & $10,5(6,8-14,2)$ \\
\hline
\end{tabular}

IC: intervalo de confiança problema pré-existente. Dentre as opções mais relatadas destacam-se as leucorreias $(50,9 \%)$, seguidas por outras queixas ginecológicas $(30,9 \%)$, doenças sexualmente transmissíveis (7\%), gestação (5\%), afecções de trato urinário (4\%) e queixas álgicas (2\%).

\section{DISCUSSÃO}

Com o aumento previsto para a incidência dos cânceres, ações direcionadas à abordagem ampliada destas patologias, englobando desde a sua detecção precoce em grupos de maior vulnerabilidade até o controle dos efeitos provenientes do seu tratamento, vêm recebendo especial destaque na agenda da saúde. Atualmente, as neoplasias são encaradas como pertencentes ao grupo de doenças crônicas, já que os avanços obtidos promoveram sensível melhora na sobrevida dos pacientes ${ }^{8,19}$. Porém, em alguns grupos estes avanços ainda não foram totalmente incorporados, tanto por questões relacionadas ao acesso aos serviços quanto à realização dos exames de detecção precoce. Conforme já mencionado, para o câncer de colo de útero estas questões têm ainda maior importância devido à sua incidência mais elevada em grupos menos favorecidos economicamente ${ }^{20}$. Diante deste cenário, estudos que possam contribuir para a captação adequada das usuárias para a realização da colpocitologia oncótica se mostram oportunos e até mesmo necessários.

Tabela 3. Perfil de realização da colpocitologia oncótica em mulheres residentes na área de cobertura da Estratégia de Saúde da Família, Nova Iguaçu, 2009

$\begin{array}{lcc}\text { Característica } & \mathbf{n} & \text { Prevalência (IC95\%) } \\ \text { Já realizaram o exame } & 281 & \\ \text { Sim } & & 95,37(92,90-97,84) \\ \text { Não } & & 4,62(2,15-7,09)\end{array}$

Frequência do exame nos últimos 12 meses

281

$\begin{array}{lc}\text { Nenhum } & 33,80(28,24-39,37) \\ 1 & 55,16(49,30-61,01) \\ 2 & 11,03(7,34-14,71)\end{array}$

Intervalo de tempo entre o último exame (anos)

257

$<1$

$72,37(66,87-77,87)$

$1-3$

$22,56(17,42-27,71)$

$>3$

$5,05(2,36-7,75)$

Realização no Sistema Único de Saúde

Sim

$72,01(66,60-77,42)$

Não

$27,98(22,57-33,39)$

Motivo da busca pelo exame

Rotina

$79,71(74,98-84,44)$

Problemas pré-existentes

IC: intervalo de confiança 
O perfil sociodemográfico da amostra avaliada abrange riscos elevados para o desenvolvimento do câncer do colo do útero. De acordo com algumas pesquisas, a faixa etária de maior prevalência da doença é a de 30 a 49 anos $^{5,6}$. Também merece destaque o maior predomínio na amostra de mulheres pardas e negras, com pouco tempo de estudo e em classes econômicas mais baixas, aspectos já mencionados como envolvidos com maior incidência deste câncer. Além disso, estas mulheres podem encontrar ainda mais dificuldade para agendamento dos exames, por estarem em plena fase laborativa e reprodutiva. Este grupo acaba por não ser absorvido pelas estratégias de captação e buscas ativas realizadas rotineiramente pelas equipes de saúde, que ainda não disponibilizam horários alternativos ${ }^{9,21}$.

Em relação ao perfil ginecológico e obstétrico da amostra, chama atenção a prevalência significativa de mulheres que relataram a utilização de anticoncepcionais hormonais orais por tempo igual ou superior a dez anos. Embora não exista um consenso sobre as consequências do uso destes medicamentos em relação à gênese do câncer do colo uterino, alguns trabalhos apontam que a anticoncepção hormonal pode acarretar aumento de infecções pelo Papiloma Vírus Humano $(\mathrm{HPV})^{22}$. O crescimento da prevalência desta infecção coincide historicamente com a ampliação dos métodos de contracepção hormonal e com a redução da adesão aos métodos de barreira, em especial a utilização de preservativos durante a atividade sexual ${ }^{8,14}$.

Outro ponto de interesse seriam os dados referentes ao número de gestações e o período de início das atividades sexuais, também considerados fatores de risco tanto para o desenvolvimento do câncer do colo uterino, quanto para a infecção pelo $\mathrm{HPV}^{22}$. Ações de promoção da saúde que contemplem a participação social, como a ESF, parecem auxiliar na melhoria destes fatores condicionantes. Rocha e Soares ${ }^{23}$, em uma pesquisa sobre o impacto das práticas comunitárias, apontam que a ESF após uma média de oito anos de atuação é capaz de reduzir significativamente a taxa de fecundidade e aumentar a empregabilidade da população adscrita. Em contrapartida, acredita-se que estas intervenções devam ser formuladas de maneira cautelosa, avaliando a dinâmica de funcionamento das famílias, o cenário histórico e social do território.

Foi encontrado um número adequado de exames realizados em Nova Iguaçu, semelhante a outros estudos no âmbito da ESF, como na cidade de Londrina $(\mathrm{PR})^{24}$. Estes números são superiores aos encontrados na maior parte das capitais brasileiras avaliadas pelo INCA, onde ainda não havia cobertura expressiva da $\mathrm{ESF}^{19}$. As características da atenção oferecida pela Saúde da Família podem representar um componente fundamental para a diferença nestes achados, já que o modelo parece favorecer o processo de captação das usuárias para a realização de práticas preventivas, a partir do trabalho em território definido e adscrição de clientela ${ }^{13}$.

Corroborando para a adesão ao método de rastreio, observa-se que o principal motivo para a procura pela colpocitologia foi a realização do exame por rotina. Este dado também foi encontrado como principal motivo de busca em outros estudos que contemplavam áreas com cobertura da Saúde da Famíliaa ${ }^{25}$. Ramos et al. ${ }^{26}$ sugerem que quanto maior o tempo de cadastro das mulheres na estratégia, maior a adesão e utilização das ações de rastreio colpocitológico. Outras ações individuais de promoção de saúde acabam sendo incorporadas pelas usuárias, gerando maior possibilidade de prevenção de outros agravos e doenças não transmissíveis. As pacientes, quando adequadamente sensibilizadas, acabam motivando seus familiares e conhecidos, funcionando como catalisadoras da comunidade. Nesta perspectiva, as ações desenvolvidas no âmbito da ESF podem trazer um diferencial fundamental no controle das doenças que mais atingem a população feminina atualmente.

Chama atenção a elevada frequência de realização do exame de rastreio da doença, que excede ao recomendado pelo Ministério da Saúde ${ }^{8}$. Embora este dado possa indicar uma boa adesão aos métodos de rastreio, acredita-se que também pode revelar certa iniquidade no acesso. Apesar de o número de exames ser razoavelmente satisfatório, algumas mulheres que habitualmente têm dificuldades e fatores de impedimento, com relação à realização dos métodos preventivos, permanecem com acesso limitado a estas práticas, enquanto outros grupos acabam realizando o exame mais vezes.

Como estratégia de enfrentamento desta problemática, aponta-se o exemplo do Programa de Seleção Coordenada, cujo objetivo é reduzir os custos e aumentar a cobertura de rastreio do câncer do colo uterino em populações de maior risco. Este programa almeja a redução da quantidade de exames em mulheres cujo esfregaço vaginal acusa normalidade e a ampliação da cobertura para aquelas que possuíam indicação da colpocitologia, em especial as com mais de 50 anos. Como resultado desta reprogramação dos serviços, observa-se diminuição da incidência de câncer invasivo em cerca de $20 \%{ }^{27}$.

A situação atual do município estudado e de todo o estado é de expansão de cobertura da ESF, o que favorece a avaliação das práticas já existentes e novas programações na busca de diagnósticos específicos para cada localidade. Os resultados evidenciam a necessidade de repensar a maneira com que as equipes de saúde da família está realizando práticas de busca ativa para a realização dos exames de rastreio da doença. Uma possibilidade seria a intensificação do exercício de vigilância em saúde e participação social em nível local, a fim de avaliar regularmente 
as ações e nortear a (re) programação das agendas e atividades. Além disso, aponta-se para a necessidade da efetivação do rastreio sistematizado, já recomendado pelo Ministério da Saúde ${ }^{8}$, a fim de contribuir para a redução dos exames desnecessários e o aumento da cobertura nos grupos de maior vulnerabilidade.

Devido ao desenho transversal do estudo, não foi possível realizar inferências causais com relação à utilização dos serviços de rastreio e as ações desenvolvidas pela ESF, demonstrando-se necessária esta investigação em pesquisas posteriores. Outros estudos já estão em andamento, a fim de contemplar os fatores impeditivos do acesso aos exames de rastreio e a sua qualidade no município estudado.

Com vistas à redução das limitações desta pesquisa, em especial pela dificuldade de adesão de participantes em inquéritos domiciliares, optou-se pela realização das entrevistas pelos ACS. Estes profissionais têm maior vínculo com a população usuária dos serviços e, na maior parte das vezes, são eles que realizam as buscas ativas para as atividades da Saúde da Família, o que pode ter contribuído para a maior participação ao estudo, bem como para os relatos mais próximos da realidade da comunidade. $\mathrm{O}$ envolvimento destes profissionais também colaborou de maneira favorável na elaboração da logística do trabalho de campo, uma vez que foi possível traçar estratégias de captação para entrevistas em horários adequados para cada uma das selecionadas.

Os esforços da ESF, no que tange às práticas de rastreio do câncer do colo uterino, contemplam minimamente o intervalo entre a realização dos exames colpocitológicos, conforme o recomendado pelo Ministério da Saúde. Entretanto, o perfil de exposição aos fatores de risco da população e a quantidade excessiva de exames realizados no ano anterior apontam para lacunas na qualidade das ações preventivas. Vale ressaltar que este cenário pode estar acontecendo em outros municípios de porte semelhante, já que a expansão e os objetivos da estratégia ainda estão longe de serem alcançados em sua plenitude no estado do Rio de Janeiro.

A Saúde da Família como estratégia norteadora da Atenção Primária tem atribuições que vão muito além da realização de exames e da prática clínica propriamente dita. Pretende oferecer aos usuários cadastrados ações que transformem a prática diária, a partir da participação mais ativa nas suas escolhas, em comportamentos saudáveis e impactem de maneira positiva os indicadores de saúde avaliados. Estes objetivos não são de fácil execução e precisam de comprometimento e parcerias mais próximas entre os serviços e seus usuários, além de apoio intersetorial e dos gestores.

A ESF é uma realidade relativamente recente no município de Nova Iguaçu, e sua cobertura vem crescendo no passado recente, acompanhando um movimento estadual. Ainda é preciso certo tempo e amadurecimento para a incorporação de suas práticas inovadoras entre os usuários, a rede de serviços e os profissionais envolvidos e avaliações locais se apresentam como importantes ferramentas na busca desta adequação.

\section{REFERÊNCIAS}

1. Ramos C, Carvalho JEC, Mangiacavalli MASC. Impacto e (i)mobilização: um estudo sobre campanhas de prevenção ao câncer. Ciênc Saúde Coletiva. 2007;12(5):1387-96.

2. Brasil. Ministério da Saúde. Secretaria de Atenção à Saúde. Instituto Nacional do Câncer (INCA). Estimativas 2010: incidência de câncer no Brasil. Rio de Janeiro: INCA; 2009.

3. Caetano R, Vianna CMM, Thuler LC, Girianelli V. Custo-efetividade no diagnóstico precoce do câncer do colo uterino no Brasil. Physis. 2006;16(1):99-118.

4. Martins LFL, Thuler LCS, Valente JG. Cobertura do exame de Papanicolaou no Brasil e seus fatores determinantes: uma revisão sistemática da literatura. Rev Bras Ginecol Obstet. 2005;27(8):485-92.

5. Organización Mundial de la Salud. Control integral del cáncer cervicouterino: guía de prácticas esenciales. Genebra: OMS; 2007.

6. Krivak TC, McBroom JW, Elkas JC. Câncer cervical e vaginal. In: Berek JE, editor. NOVAK Tratado de Ginecologia. Rio de Janeiro: Guanabara Koogan; 2005. p. 1119-62.

7. Brito CMS, Nery IS, Torres LC. Sentimentos e expectativas das mulheres acerca da citologia oncótica. Rev Bras Enferm. 2007;60(4): 387-90.

8. Brasil. Ministério da Saúde. Secretaria de Atenção à Saúde. Instituto Nacional do Câncer. Prevenção do câncer do colo do útero: manual técnico - profissionais de saúde. Rio de Janeiro: INCA; 2002.
9. Rafael RMR, Moura ATMS. Barreiras na realização da colpocitologia oncótica: um inquérito domiciliar na área de abrangência da Saúde da Família de Nova Iguaçu, Rio de Janeiro, Brasil. Cad Saúde Pública. 2010;26(5):1045-50.

10. Greenwood SA, Machado MFAS, Sampaio NMV. Motivos que levam mulheres a não retornarem para receber o resultado de exame Papanicolaou. Rev Latino-Am Enferm. 2006;14(4):503-9.

11. Ronzani TM, Silva CM. O Programa Saúde da Família segundo profissionais de saúde, gestores e usuários. Ciênc Saúde Coletiva. 2008;13(1):23-34.

12. Costa EMA, Carbone MH. Saúde da família - uma abordagem interdisciplinar. Rio de Janeiro: Rubio, 2004

13. Brasil. Ministério da Saúde. Política Nacional de Atenção Básica. Brasília: Ministério da Saúde; 2006.

14. Brasil. Ministério da Saúde. Secretaria de Vigilância em Saúde. Instituto Nacional do Câncer. Prevenção do câncer do colo do útero: manual técnico - organizando a assistência. Rio de Janeiro: INCA; 2002.

15. Instituto Brasileiro de Geografia e Estatística (IBGE). Pesquisa nacional por amostra de domicílios: questionário da pesquisa. Rio de Janeiro; 2006.

16. Associação Brasileira de Empresas de Pesquisa (ABEP). Critério de Classificação Econômica Brasil [Internet]. 2011 [cited 2008 Dec 02]. Available from: http://www.abep.org/novo/Content.aspx?ContentID=139 
17. Brasil. Ministério da Saúde. Secretaria de Vigilância em Saúde. Instituto Nacional do Câncer. Questionário individual - tipo A. Inquérito domiciliar sobre comportamentos de risco e morbidade referida de doenças e agravos não transmissíveis. Rio de Janeiro: INCA; 2002.

18. Rodrigues L, Werneck GL. Estudos caso-controle. In: Medronho RA, Bloch KV, Luiz RR, Werneck GL. Epidemiologia. São Paulo: Atheneu; 2009.

19. Brasil. Ministério da Saúde. Secretaria de Vigilância em Saúde. Instituto Nacional de Câncer. Inquérito domiciliar sobre comportamentos de risco e morbidade referida de doenças e agravos não transmissíveis: Brasil, 15 capitais e Distrito Federal, 2002-2003. Rio de Janeiro: INCA; 2004.

20. Novaes HMD, Braga PE, Schout D. Fatores associados à realização de exames preventivos para câncer nas mulheres brasileiras, PNAD 2003. Ciênc Saúde Coletiva. 2006;11(4):1023-35.

21. Pinho AA, França-Junior I. Prevenção do câncer de colo do útero: um modelo teórico para analisar o acesso e a utilização do teste de Papanicolaou. Rev Bras Saude Mater Infant. 2003;3(1):95-112.

22. Caldas I, Teixeira SM, Rafael RMR. O papilomavírus humano como fator preditor do câncer do colo uterino: estudo de atualização sobre as ações preventivas de enfermagem. Rev Enferm UFPE. 2010;4(2):831-9.
23. Rocha R, Soares RR. Evaluating the impact of community-based health interventions: evidence from Brazil's Family Health Program. 2010;19:126-58.

24. Silva IMR, Brenna SMF, Moriwaki OM, Mariani Neto C. Avaliação dos programas brasileiros para controle do câncer genital feminino. Rev Adm Saúde. 2004; 6(24):97-102.

25. Oliveira MMHN, Silva AAM, Brito LMO, Coimbra LC. Cobertura e fatores associados à não realização do exame preventivo de Papanicolaou em São Luís, Maranhão. Rev Bras Epidemiol. 2006;9(3):325-34.

26. Ramos AS, Palha PF, Costa Júnior ML, Sant'Anna SC, Lenza NFB. Perfil de mulheres de 40 a 49 anos cadastradas em um núcleo de saúde da família, quanto à realização do exame preventivo de Papanicolaou. Rev Latinoam Enferm. 2006;14(2):170-4.

27. Nygard JF, Skare GB, Thoresen S. The cervical cancer screening programme in Norway, 1992-2000: changes in pap smear coverage and incidence of cervical cancer. J Med Screen. 2002;9(2):86-91.

Recebido em: 13/02/2012

Aprovado em: 04/07/2012 